\title{
Model and control of simulated respiration for animation
}

\author{
Victor B. Zordan, Bhrigu Celly, Bill Chiu, and Paul C. DiLorenzo* \\ University of California Riverside
}
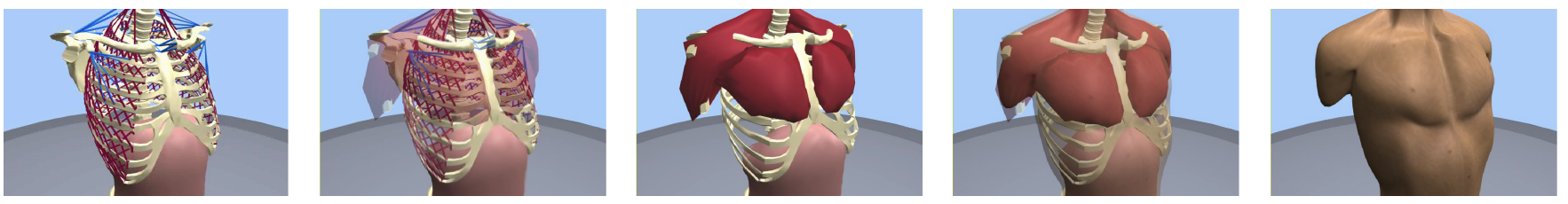

Breathing is a critical body function and its motion is a telltale signature for the living. However, in animation, respiration and its deformation of the torso have remained stylistic and are often overly simple or ignored entirely. We present an anatomically inspired, physically based model of the human torso for the visual simulation of respiration using a mixed system of rigid and deformable parts. Our system combines a custom deformable simulation system, that preserves volume based on pressure, with an available rigid-body dynamics solver.

The visual motion of human breath is derived from two actively moving muscle groups - the diaphragm and the intercostal muscles attached to the ribs. These two active components lead to the movement of the chest, shoulder, arms and abdomen and even, through the spine, the involuntary motion of the head associated with breath. In the ribcage, the inner and outer intercostal muscles between the ribs change the shape of the ribcage overall and drive passive deformations of many of the chest and back's muscles. The diaphragm works with the rib muscles to expand the lung cavity. The movement of the abdomen wall surrounding the gut is indirectly driven by the pumping of the diaphragm and stores potential energy through inhalation to reset the diaphragm during exhalation. For more on respiratory physiology, see Mines [1993].
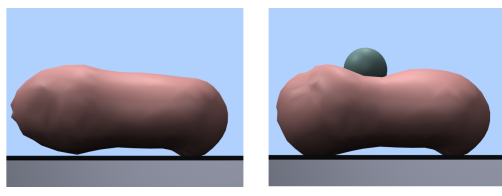

Figure 1: Simulated impact of constant volume gut.

Our breathing system exclusively uses forces, computed from simple spring-muscles elements, to drive the movement of rigid and deformable components. And, through realistic insertion points these non-compressive muscles elements (that pull, but not push,) help to constrain the possible movements and yield an easily controlled rigid-body system for the ribcage. We synthesize the motion of the abdomen wall by modeling the gut as a deformable, incompressible volume as seen in Figure 1. For deformation, the system computes the associated spring-muscle forces from the abdomen and diaphragm muscle groups and applies them to a distribution of point masses placed at the spring intersections. With volume preservation, the gut-body simulation deforms through a balance of surface tension and internal pressure forces, emulating the physical nature of the human gut as it moves during breath. More on anatomically based volumes is described by Teran et al. [2003].

We control activation for muscle groups by changing the relative contraction as a time-varying parameter based on the frequency of the desired breath. The intercostals and diaphragm are con-

*e-mail: vbz | bcelly | bill | pdiloren@cs.ucr.edu trolled uniquely based on their neural pathways' differing connection points with the spine [Mines 1993] and, as such, we supply two unique patterns for the rib and diaphragm contraction. For the diaphragm, often referred to as a pump or a plunger, a step function created the desired response. For the intercostals, out-of-phase sine curves lead to visually pleasing, smooth oscillations for the ribcage motion.

We present a high-level view of a more detailed implementation of breathing focusing on the concepts of modeling breath which are quite independent of the type of model or simulation. More details and animations can be found at our website (www.cs.ucr.edu/rgl/breath.html). In addition, at a fundamental level, we wish to revitalize the biological importance of deformations of the trunk which, in its purest form is a living and integral component of the whole body. Through this methodology, we have shown that several hundred unique string-like muscles elements, like those of the intercostals, can act in unison based on simple activation inputs to perform complex movements for the overall change in shape of the ribcage during breath, for example. We anticipate that this work will entice other researchers to consider the physically modeling of the human body from the inside out, based on anatomical form.

\section{References}

Mines, A. H. 1993. Respiratory Physiology. Raven Press.

Teran, J., Blemker, S., Hing, V. N. T., And Fedkiw, R. 2003. Finite volume methods for the simulation of skeletal muscle. In ACM SIGGRAPH Symposium on Computer Animation, 68-74.

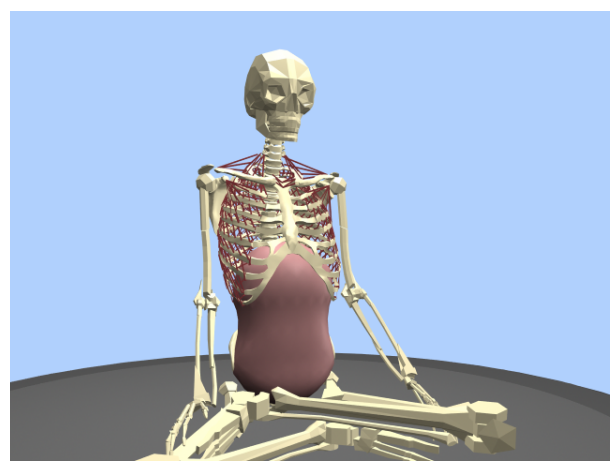

Figure 2: Skeleton with rigid-body (secondary) elements for the shoulders and arms. Deformable secondary elements are also added for the pectorals and shoulders, seen in top middle image. 